\title{
Françoise Bourquelot, L'homme et la betterave à sucre
}

Association Radio Valois Multien, 2007, 234 p., $12 €$

\section{Nicole Mathieu}

\section{CpenEdition}

Journals

Édition électronique

URL : http://journals.openedition.org/economierurale/2187

DOI : $10.4000 /$ economierurale.2187

ISSN : 2105-2581

Éditeur

Société Française d'Économie Rurale (SFER)

Édition imprimée

Date de publication : 5 avril 2009

Pagination : 92-93

ISSN : 0013-0559

Référence électronique

Nicole Mathieu, «Françoise Bourquelot, L'homme et la betterave à sucre », Économie rurale [En ligne], 310 | Mars-avril 2009, mis en ligne le 06 novembre 2009, consulté le 21 septembre 2020. URL : http:// journals.openedition.org/economierurale/2187; DOI : https://doi.org/10.4000/economierurale.2187 
Françoise BOURQUELOT

\section{L'homme et la betterave à sucre}

Association Radio Valois Multien, 2007, 234 p., $12 €$

$\mathbf{P}$ ublié par une association, la Radio du Valois et du Multien - du nom d'une région agricole qui revit depuis que, il y a 15 ans, les collectivités locales vantent la richesse historique de cette région : Les Valois, Jeanne-d'Arc, une nuit dans le vieux Crépy, la beauté des 35 clochers de la vallée de l'automne, pour développer le tourisme en hausse constante - ce livre', destiné à être de diffusion locale, mérite d'être chaleureusement recommandé à des lecteurs pour sa valeur narrative et en même temps universelle.

Reprenant le principe des titres de la collection de Géographie humaine dirigée par Pierre Deffontaines ${ }^{2}$ cette courte et " modeste monographie sur la filière du sucre de betterave » se lit d'un trait tant il nous emporte dans une saga où l'histoire de la culture de la betterave à sucre et de sa transformation, depuis l'époque napoléonienne jusqu'à nos jours, constitue le scénario et dont les acteurs - aussi présents que dans Les raisins de la colère - sont les salariés agricoles (saisonniers et permanents), les grands agriculteurs de ce pays ainsi que les ouvriers, le patron fondateur et sa famille d'une des sucreries locales (Vauciennes) qui, née d'un capitalisme familial, emportée dans la mondialisation, disparaît en 1999.

Ce coup de cœur pour L'homme et la betterave à sucre va au-delà d'une nostalgie pour un mode d'écriture : la simplicité du récit et l'empathie pour ceux dont on narre I'histoire ; au-delà aussi d'une préférence pour ce mode de connaissance, les enquêtes sur les familles et les régions à la façon des

1. Pour commander le livre: RVM, 10 Place JeanPhilippe Rameau, 60800 Crépy-en-Valois. Prix : $12 €$ plus $1,75 €$ de frais de port.

2. Dont le célèbre L'homme et la charrue à travers le monde de A. G. Haudricourt et M. JeanBrunhes Delamarre mais aussi L'homme et la forêt ou L'homme et l'hiver au Canada de Pierre Deffontaines.
Ouvriers européens et l'ingénierie sociale de Frédérique Leplay. Plaisir de l'intelligence que cette sorte de monographie procure parce qu'elle vise à ne pas perdre les traces des travaux et des jours, des gestes et des émotions de la quotidienneté, parce qu'elle est exercice de mémoire, non pour exacerber l'esprit victimaire mais pour redonner vie précise et exacte aux gens qui subissent et rusent avec leur sort pour transformer la peine en joie et en biens ayant valeur économique et sociale. On y retrouve le sens de ce genre trop souvent dénigré car c'est là seulement que l'on peut s'efforcer d'observer, de décrire, d'analyser et de comprendre ce que sont les relations des hommes avec la nature et avec les lieux où ils travaillent et vivent dans leur dimension matérielle comme dans leur dimension idéelle. Qui permet aussi de réfléchir aux différences et aux ressemblances que ce récit a avec la conjoncture (phase) actuelle et, en particulier, avec la difficile articulation entre globalisation et développement durable.

L'auteure a enseigné l'économie rurale à I'EHESS et est connue pour ses analyses remarquables des " salariés agricoles » suivis depuis les années 1960 jusqu'à aujourd'hui, en particulier les saisonniers étrangers en Espagne et dans le sud de la France, en passant par les accords de Grenelle en 1968 et la lutte de Solferino dans les Landes.

Le point de départ de son livre est un projet de quinze émissions sur l'introduction de la betterave sucrière, projet accepté par I'association Radio Valois Multien dont le but est de renforcer la communication sociale entre les anciens et les nouveaux venus. II s'est construit sur l'idée de raconter aux nouveaux habitants de ces communes de l'Oise qui l'ignorent l'histoire des lieux et de la région où ils vivent. La série radiophonique s'appuie sur une enquête non directive auprès d'agriculteurs, d'anciens ouvriers agricoles souvent saisonniers restés dans la région ou repartis dans leur pays d'origine, de techniciens et de responsables syndicaux. Puis ont été menées une deuxième série d'émissions et une deuxième enquête après la fermeture en 1999 de la sucrerie de Vauciennes qui croise l'histoire de la famille qui a dirigé 
cette sucrerie pendant 125 ans et celle de ses ouvriers permanents ou saisonniers.

Comme s'il suivait cette temporalité, l'ouvrage s'organise en deux parties consacrées d'abord à " La betterave à sucre et ceux qui la cultivent » où le récit est porté par ce qui se passe dans les champs, puis au passage " De la fabrique à l'usine » qui met l'industrie en son centre. Mais au-delà de cette division, l'auteure nous engage à une véritable réflexion sur des points qui continuent de nous hanter aujourd'hui : "Connaître les raisons qui ont poussé tant d'ouvriers à quitter leur pays ; analyser les conséquences du développement technique sur la déqualification des ouvriers et la diminution des emplois ; mesurer l'influence des syndicats agricoles et ouvriers; pointer les effets de la mondialisation sur la production de betteraves sucrières et sur les sucreries; essayer de comprendre pourquoi la betterave est aujourd'hui conviée à servir de matière première à la production de bioéthanol ».

Ce récit bien ordonné se lit comme un roman tant il excelle dans l'évocation et des choses et des gens. Détail des choses d'abord : tout est minutieusement décrit qu'il s'agisse de la façon dont sont démariées, décolletées, râpées les betteraves à sucre, de la bascule sur laquelle elles sont pesées, ou du petit train à voie étroite qui les conduit du champ au bord de la route... Les outils, les machines dans l'usine, les logements des ouvriers (le dedans comme le dehors) sont décrits minutieusement et dans l'exactitude (cf. les illustrations et les photos). Mais ce fourmillement de détails n'est jamais dissocié de l'interprétation en termes de conditions de travail et de relations sociales. Il vous instruit en même temps qu'il vous projette aux côtés des faits et des gens comme s'ils étaient là, à portée de vos yeux et de votre connaissance. Il est toujours replacé dans le temps qui est le fil conducteur pour comprendre l'importance de l'évolution des techniques de l'innovation et de ce qu'elle modifie dans la condition des travailleurs et de l'économie.

Le détail des gens est encore plus évocateur et saisissant. L'histoire des migrations saisonnières et les raisons pour lesquelles les Belges, les Camberlots, les Bretons, les Polonais puis les Italiens, les Espagnols et les Portugais sont venus dans le Valois s'incarnent non seulement dans leurs dires avec des façons de parler qui diffèrent selon qu'il s'agisse d'hommes ou de femmes, de Belges ou d'Italiens, de saisonniers hantés par le retour au pays ou de ceux prêts à s'enraciner... Mais là encore les portraits et les dires ne sont jamais dissociés de la réflexion des actes, sur le travail, sur les ruses pour éviter le chômage, sur ce que font « les enfants et leur mères ", sur la façon de faire la fête selon le pays d'origine et sur le mélange qui s'en suit, bref sur les modes d'habiter des gens du lieu qu'ils soient gros agriculteurs, ouvriers permanents ou saisonniers, techniciens, syndicalistes ou patrons, sur ce qui rend un lieu habitable.

Le plaisir de lire ce livre vient aussi de cette présentation astucieuse d'une page de droite où le récit est linéaire et d'une page de gauche illustrée de photos, de textes juridiques, d'entretiens anciens faits par d'autres enquêteurs et où, surtout, s'expriment librement les gens. Morceaux d'entretiens parfois si vigoureux et justes que l'on ne peut s'empêcher de commencer par la page de gauche pour deviner et ressentir ce qui sera analysé sur la page de droite.

Il s'agit bien de méthode historique : recours aux archives de toutes sortes, aux écrits de l'époque et actuels, aux images diverses et aux récits de vie, témoignages souvent bouleversants d'étrangers, indigents cherchant à mieux vivre et à fonder une famille, évoquant à la fois la peine et les joies des travaux et des jours, regrettant le temps où I'on trouvait du travail et où la mobilisation collective et la solidarité pouvait changer le cours des choses.

Mais cette histoire locale est aussi histoire de la mondialisation car sous l'homogénéité du destin global surgissent les singularités qui font le rapport des gens aux lieux. Une fois le livre fermé on voudrait y revenir, revenir aux faits qui y sont rapportés pour les reconsidérer en fonction de l'aujourd'hui.

Nicole MATHIEU UMR Ladyss

Dynamiques sociales et recomposition des espaces - Université de Paris $X$ 